Gazi University
Journal of Science
$\mathrm{http} / /$ dergipark.gov.tr/gujs

\title{
Calculated Lattice Thermal Conductivity of Magnetite Thin Films based on Modified Callaway Model
}

\author{
Ibrahim Nazem QADER ${ }^{1,2}$ D, Ecem ONER $^{1}$ D, Mediha KOK $^{1, *}$ \\ ${ }^{I}$ Firat University, Faculty of Science, Department of Physics, Elazig, Turkey \\ ${ }^{2}$ University of Raparin, Faculty of Science, Department of Physics, Sulaymaneyah, Iraq
}

Highlights

- LTC of magnetite was studied.

- Modified Callaway Model used.

- Physical quantities were estimated.

\begin{tabular}{l} 
Article Info \\
\hline \\
Received: 13 Feb 2021 \\
Accepted: 31 Mar 2021 \\
Keywords \\
\hline $\mathrm{Magnetite}_{\mathrm{Fe}_{3} \mathrm{O}_{4}}$ \\
Thin film \\
Lattice thermal conductivity \\
Modified Callaway Model
\end{tabular}

\begin{abstract}
Thermal conductivity is an important parameter for semiconductor materials used in the nanoscale applications. In this study, the lattice thermal conductivity (LTC) of magnetite thin films was simulated by Modified Callaway Model. To fit the experimental data, some quantities, such as mean bond length, the lattice constant, and volume per atom were calculated. Also, the model is based on some other quantities, such as gruneisen parameter, electron concentration, and surface roughness that were found through fitting theoretical with experimental LTC. As a result, this model could work comparably well in all sizes, and the relationship between the fitting parameters and the thickness of the magnetite films was estimated.
\end{abstract}

\section{INTRODUCTION}

Magnetite nano-sized materials have superior physical and chemical properties due to the quantum size effect and surface effect. Recently, $\mathrm{Fe}_{3} \mathrm{O}_{4}$ paramagnetic have been extensively investigated due to their high coercivities and low Curie temperatures [1-3]. Besides, the most important feature of the magnet is that it is non-toxic [4], therefore, biosensors are used in the contrasting agent in magnetic resonance (MR) imaging and magnetic targeted-drug delivery systems $[5,6]$.

Two important factors that can transfer heat inside solid materials are free electrons and phonons. The density of free electrons in metals is extremely higher than the other elements, therefore they have a significant role to transfer heat energy through solid materials. On the other hand, phonons are formed by the vibration of atoms in solid materials that have only three degrees of freedom (DOF). Basically, the heat energy can transfer in both respective methods, however, in metals, the transfer of heat by free electrons are comparably dominant, while in insulator and semiconductor materials, phonons are the most obvious factor to transfer heat energy since the optical phonon velocity and group velocity have low and high value, respectively. Lattice thermal conductivity (LTC) is a significant parameter for some applications, such as electronic components, to overcome the exceeding heat and to enhance the device performance $[7,8]$.

The magnetic particles used by Chinese sailors and magnetotactic organisms are chemically composed of $\mathrm{Fe}_{3} \mathrm{O}_{4}$ or magnetite. "Magnetite" is named after the Magnesia region where large magnetite deposits are found in Asia Minor. Magnetite, containing both ferrous (reduced) and ferric (oxidized) iron species, is 
often described as iron II, III oxide. Magnetite is a Fe mineral ferrimagnetic material with a cubic inverse spinel structure. It is a mineral found in magnetite rocks and one of the oxides of iron. The reaction scheme of magnetite is defined as [9]:

$\underset{\text { Ferrous Oxide }}{\mathrm{FeO}}+\underset{\text { Ferric Oxide }}{\mathrm{Fe}_{2} \mathrm{O}_{3}} \rightarrow \underset{\text { Magnetite }}{\mathrm{Fe}_{3} \mathrm{O}_{4}}$

Bloch and Neel thought that magnetic nanoparticles exhibit superparamagnetic behavior due to the small strain caused by the negligible energy barrier in the hysteresis of the magnetization loop [10]. To date, costly treatment techniques such as precipitation, evaporation, solvent extraction, ion exchange, reverse osmosis have been used to remove metallic ions from wastewater. Fortunately, recent advances in the nano technique have shed light on this area [11-13]. Nanoparticles, which are generally characterized by a significant amount of surface, are of great interest due to their unique properties and potential applications. Additionally, magnetic $\mathrm{Fe}_{3} \mathrm{O}_{4}$ nanoparticles have attracted a lot of attention not only in the magnetic field but also in medical care fields in recent years [14-16].

Many researchers have applied Modified Callaway Model for single element nanowires, such as Si [17] and Ge [18]; binary nanowires, such as InAs [19], GaAs [20], and GaN [21]; thin film, such as Si [17]. In the aforementioned literature, the authors reported some important physical quantities such as dislocation density, carrier concentration, roughness, and gruneisen parameter. Also, they estimated other quantities, such as lattice parameter, melting temperature, and Debye temperature for the nano-sized specimens. Park et al. reported the lattice thermal conductivity for magnetite $\left(\mathrm{Fe}_{3} \mathrm{O}_{4}\right)$ for thin films with different thicknesses. They also tried to apply the Callaway model to fit their obtained results with the theoretical approach, however since their model is simplified of the Callaway Model, so it could not fit perfectly the data.

In this study, the thermal conductivity of magnetite thin films with diameters of 100, 300, and $400 \mathrm{~nm}$ for very low temperatures up to room temperature was modeled. The Modified-Callaway Model is based on parameters that defined for nanoscale materials. Throughout the study, surface roughness, carrier concentration, dislocation, impurity, and gruneisen parameters were also found.

\section{THEORY OF CALCULATIONS}

The Boltzmann transport equation provides phonon relaxation time approximation, which is given as follows:

$\kappa=A T^{3} \int_{0}^{\theta_{D} / T} \tau_{c} J(x) d x$

where

$A=\left(k_{B} / \hbar\right)^{3}\left(k_{B} /\left(2 \pi^{2} v\right)\right)$

$J(x)=x^{4} e^{x}\left(e^{x}-1\right)^{-2}$,

$x=\hbar \omega / k_{B} T$,

also, $k_{B}$ is the Boltzmann constant $\left(=1.38065 \times 10^{-23} \mathrm{~m}^{2} \cdot \mathrm{kg} \cdot \mathrm{s}^{-2} \cdot \mathrm{K}^{-1}\right)$, and $\hbar$ is the reduced Planck constant $\left(1.05457 \times 10^{-23} \mathrm{~J} \cdot \mathrm{s}\right)$. Moreover, $\tau_{c}$ is the combination of phonon scattering; $v$ is group velocity of acoustic phonon; $\omega$ is the angular frequency; $\theta_{D}$ and $T$ are the Debye temperature (at this temperature, all bases in the lattice usually reach their highest frequency, after which the absorbed heat causes the material to expand and melt) and absolute temperature, respectively. 
Asen- Palmer et al. [22] improved the Debye-Callaway model proposed for calculating LTC as a function of temperature, dimension, and size of those materials that phonons are the dominant factor for transferring heat energy. The model provides three-phonon processes of the total of one longitudinal $\left(\kappa_{L}\right)$ and two transverse $\left(\kappa_{T}\right)$ phonons $\left(\kappa=\kappa_{1}+\kappa_{2}\right)$. The total LTC is given as follows [23]:

$\kappa=\kappa_{L}+2 \kappa_{T}$,

where

$\kappa_{L}=\kappa_{L_{1}}+\kappa_{L_{2}}$

$\kappa_{T}=\kappa_{T_{1}}+\kappa_{T_{2}}$,

where $\mathrm{L}$ and $\mathrm{T}$ indicate longitudinal and transverse. The longitudinal terms in Equation (7) can be written as [17]:

$\kappa_{L_{1}}=\frac{1}{3} A_{L} T^{3} \int_{0}^{\theta_{D}^{L}} \tau_{c}^{L}(x) J(x) d x$

$\kappa_{L_{2}}=\frac{1}{3} A_{L} T^{3}\left[\int_{0}^{\theta_{D}^{L}} \frac{\tau_{c}^{L}(x)}{\tau_{N}^{L}(x)} J(x) d x\right]^{2}\left[\int_{0}^{\theta_{D}^{L}} \frac{\tau_{c}^{L}(x)}{\tau_{N}^{L}(x) \tau_{R}^{L}(x)} J(x) d x\right]^{-1}$.

Similarly, the transverse terms are given in Equation (8), can be obtained from the equations in [17]:

$\kappa_{T_{1}}=\frac{1}{3} A_{T} T^{3} \int_{0}^{\theta_{D}^{T}} \tau_{c}^{T}(x) J(x) d x$

$\kappa_{T_{2}}=\frac{1}{3} A_{T} T^{3}\left[\int_{0}^{\theta_{D}^{T}} \frac{\tau_{c}^{T}(x)}{\tau_{N}^{T}(x)} J(x) d x\right]^{2}\left[\int_{0}^{\theta_{D}^{T}} \frac{\tau_{c}^{T}(x)}{\tau_{N}^{T}(x) \tau_{R}^{T}(x)} J(x) d x\right]^{-1}$,

where the term of $A_{T(L)}$ in Equations (11) and (12) can be defined as the following equation in [19]:

$A_{T(L)}=\left(\frac{k_{B}}{\hbar}\right)^{3} \frac{k_{B}}{2 \pi^{2} v_{T(L)}}$

where $v$ is the group velocity of acoustic phonon. A photon scatters in the material through different types, including phonon-phonon (or normal) scattering, $\tau_{N}^{L(T)}$; inharmonic interaction or Umklapp three-phonon scattering, $\tau_{U}^{L(T)}$; phonon-impurity, $\tau_{M}^{L(T)}$; phonon-electron, $\tau_{p h-e}^{L(T)}$; phonon-boundary, $\tau_{B}^{L(T)}$; and phonondislocation $\tau_{D C}^{L(T)}$ scattering, where the superscript indicates longitudinal or transverse. The combination of all respective photon scattering scenarios is given as [24]:

$\left(\frac{1}{\tau_{C}^{L(T)}}\right)=\left(\frac{1}{\tau_{N}^{L(T)}}\right)+\left(\frac{1}{\tau_{U}^{L(T)}}\right)+\left(\frac{1}{\tau_{M}^{L(T)}}\right)+\left(\frac{1}{\tau_{B}^{L(T)}}\right)+\left(\frac{1}{\tau_{p h-e}^{L(T)}}\right)+\left(\frac{1}{\tau_{D C}^{L(T)}}\right)$

The Umklapp scattering (U-process) rate modes given in Equation (14) can be obtained as follows [20]:

$$
\left[\tau_{U}^{L(T)}\right]^{-1}=B_{U}^{L(T)}\left(\frac{k_{B}}{\hbar}\right)^{2} x^{2} T^{3} e^{-\left(\theta_{D}^{L(T)} / 3 T\right)}
$$


where the Umklapp parameter strength $B_{U}^{L(T)}$ reversely related to the Debye temperature:

$B_{U}^{L(T)}=\frac{\hbar \gamma_{L(T)}^{2}}{M v_{L(T)}^{2} \theta_{D}^{L(T)}}$

The Gruneisen parameter, $\gamma_{L(T)}$, explains how changing the volume of a crystal can influence the vibrational behavior [25]. The $\gamma_{L(T)}$ can be found by fitting theoretical LTC with the corresponding experimental data. In Equation (16), $M$ is the average atomic mass in the crystal, which is equal to $\rho \times V$, where $\rho$ is the density and $V$ is lattice volume. The value of $\mathrm{M}$ obtained for magnetite is $5.49241 \times 10^{-26}(\mathrm{~kg})$. The Debye temperature given in Equation (15) can be obtained by [26]:

$\theta_{D}^{L(T)}=\left(\frac{6 \pi^{2}}{V}\right)^{1 / 3} \frac{\hbar v_{L(T)}}{k_{B}}$

The phonon-phonon scattering is a process, whereby two phonons interact with each other and produce a new phonon that has the sum of both phonons' momentum vectors. It can be achieved by using the following formula [27]:

$\left[\tau_{N}^{L(T)}\right]^{-1}=B_{N}^{L(T)} \omega^{2} T^{3}$

where $B_{N}$ is the normal modes in the strength parameter for both modes (transverse and longitudinal). Equation (18) can be extended as follows:

$B_{N}^{L}=\frac{k_{B}^{3} \gamma_{L}^{2} V}{M \hbar^{3} v_{L}^{5}}$ and $B_{N}^{T}=\frac{k_{B}^{4} \gamma_{T}^{2} V}{M \hbar^{3} v_{T}^{5}}$

Another scattering is the phonon- isotope $\left(I_{\text {iso }}\right)$ scattering. Since isotope elements have a different number of neutrons, so their atomic mass is slightly different from one species to another, therefore in a specific temperature, the isotopes have a different vibration, which causes a scattering termed phonon-isotope scattering. Additionally, materials probably have impurities, which are different from that of host atoms, thus they can scatter phonons. This process is called the phonon- impurity $\left(I_{i m p}\right)$ scattering rate that can be found in terms of the dimensionless variable $x$ [28]:

$\left[\tau_{M}^{L(T)}\right]^{-1}=\left(I_{i s o}^{L(T)}+I_{i m p}^{L(T)}\right) \omega^{4}$

where

$I_{i s o}^{L(T)}=\frac{V \Gamma}{4 \pi v_{L(T)}^{3}}$,

and

$I_{i m p}^{L(T)}=\frac{3 V^{2} S^{2}}{\pi v_{L(T)}^{3}} N_{i m p}$

where $V$ is the lattice volume; $N_{\text {imp }}$ refers to the concentration of all kind of impurities; $\mathrm{S}$ is the scattering factor which is ordinarily equal to one. Also, $\Gamma$ represents the strength of the mass difference scattering, which for a binary compound can be obtained by [27]: 
$\Gamma(\mathrm{AB})=2\left[\left(\frac{M_{A}}{M_{A}+M_{B}}\right)^{2} \Gamma(\mathrm{A})+\left(\frac{M_{B}}{M_{A}+M_{B}}\right)^{2} \Gamma(\mathrm{B})\right]$,

where $\Gamma(\mathrm{AB})$ is the mass difference scattering for the binary compound; $A$ and $B$ are the constituents, which are $\mathrm{Fe}$ and $\mathrm{O}$ for this study; $M_{A}$ and $M_{B}$ are average atomic mass for element $\mathrm{A}$ and $\mathrm{B}$, respectively; $\Gamma(\mathrm{A})$ and $\Gamma(B)$ are the strength of mass difference scattering for the $A$ and $B$ components, which can be obtained by:

$\Gamma=\sum_{i} c_{i}\left(\frac{m_{i}-\bar{m}}{\bar{m}}\right)^{2}$

where $c_{i}$ is the abundance rate of element $i ; m_{i}$ and $\bar{m}$ are the atomic mass of the $i^{\text {th }}$ isotope and average atomic mass of an element, respectively. The average atomic mass was calculated by [27]:

$\bar{m}=\sum_{i} c_{i} m_{i}$

Equation (22) also can be used to find $M_{A}$ and $M_{B}$ given in Equation (20). The calculated value of $\bar{m}$ for iron and oxygen are 55.8451 and $15.9993 \mathrm{amu}$, respectively. Besides, by substituting the respective parameters into Equation (20) the value of $\Gamma(\mathrm{AB})$ was obtained as 0.00549716 .

The phonons can be scattered by the boundaries, which is termed as phonon-boundary scattering rate that can be expressed as:

$\left[\tau_{b}^{L(T)}(L)\right]^{-1}=v_{L(T)} / d$

where $d$ is the effective diameter of the $\mathrm{Fe}_{3} \mathrm{O}_{4}$ thin films that was adjusted to adequate the LTC. In nanocrystal materials, the phonon-boundary scattering rate is independent of the acoustic group velocity, effective diameter $\left(L_{e f f}\right)$, temperature, and frequency [29]:

$\left[\tau_{b}^{L(T)}(L)\right]^{-1}=\frac{v_{L(T)}}{L_{e f f}}=v_{L(T)}\left(\frac{1}{L_{c}}+\frac{1}{L}\right)$

where $L$ is known as the length of a nanowire or thickness of a thin film specimen. The value of $L_{e f f}$ depends on the temperature, whereby, for a temperature less than Debye temperature, it can get a value comparable with the cross-sectional of the sample, which is called Casimir length $\left(L_{c}\right)$. Equation (24) can be rewritten as:

$\left[\tau_{b}^{L(T)}(L, \varepsilon)\right]^{-1}=v_{L(T)}\left(\frac{1}{L_{c}} \frac{(1-\varepsilon)}{(1+\varepsilon)}+\frac{1}{L}\right)$

where $1 / L_{e f f}$ is specularity parameter; $\varepsilon$ is the surface roughness rate that has a value between 0 and 1 . A phonon can diffuse ideally when the surface is smooth $(\varepsilon=0)$, on the other hand, it can diffuse from the surface completely (specular reflection) for $\varepsilon=1$.

Normally all crystalline materials, including semiconductors, have dislocations, that can make a restriction in front of flowing phonons. Phonons can scatter from the core of dislocation lines and/or by the elastic strain field on the dislocation lines. The phonon-dislocation rate can be obtained by [30]:

$\left[\tau_{D C}^{L(T)}(x)\right]^{-1}=\eta N_{D} \frac{V_{o}^{4 / 3}}{v_{L(T)}^{2}}\left(\frac{k_{B} T}{\hbar}\right)^{3} x^{3}$, 
where the density of the dislocation is represented by $N_{D}$, the weight factor is given by $\eta$, which is a mutual tendency of dislocation lines and $\Delta T$ (temperature gradient) and its value is equal to 0.55 [31].

Electrons play an important role in transferring heat energy inside solid materials, however, they can scatter phonons that move through a material. The acoustic phonons can be scattered in both longitudinal and transverse direction that is given by [32]:

$\left[\tau_{p h-e}^{L(T)}(x)\right]^{-1}=\frac{n_{e} E^{2} x}{\rho v_{L(T)}^{2} \hbar} \sqrt{\frac{\pi m^{*} v_{L(T)}^{2}}{2 k_{B} T}} \times \exp \left(-\frac{m^{*} v_{L(T)}^{2}}{2 k_{B} T}\right)$,

where $n_{e}$ is a fitting parameter in this model that represent the concentration density of conduction electron; $E$ is the deformation potential, which is equal to 0.16 of electron charge for magnetite [33]; $\rho$ is mass density, which for $\mathrm{Fe}_{3} \mathrm{O}_{4}$ is $5170 \mathrm{~kg} \cdot \mathrm{m}^{-3}$ [34]; $\mathrm{m}^{*}$ is the effective mass of electron that is equal to $100 \mathrm{me}_{e}$ [35]. The mass density has not a constant value for nanomaterials, since the mean bond length is changed by changing the size of the nano-sized materials, and hence the volume per atom (V) is also changed, which influence the value of the mass density such that [36]:

$\rho(r)=\frac{\rho(\infty) V(\infty)}{V(r)}$

where the density for bulk material (materials with more than hundreds of nanometers on each side) is given by $\rho(\infty)$, also the volume per atom for the bulk material is represented by $V(\infty)$. By using Equation (28) the value of mass density for each magnetite thin films were found.

Besides, the value of sound group velocity is also a function of the size of nano-material, $v(r)$, and its value can be obtained by the following relation [37]:

$\frac{v(r)}{v(\infty)}=\frac{\theta_{D}(r)}{\theta_{D}(\infty)}$

where $\theta(\infty)$ is the bulk Debye temperature and $\theta(r)$ is the Debye temperature as a function of temperature. The Debye temperature for bulk material in both longitudinal and transverse was found by Equation (17). The size-dependent Debye temperature can be calculated by:

$\left(\frac{\theta_{D}(r)}{\theta_{D}(\infty)}\right)^{2}=\frac{T_{m}(r)}{T_{m}(\infty)}$

where $v(\infty)$ is group velocity that is assumed as a constant value for bulk magnetite. The value of group velocity for longitudinal and transverse magnetite are 3263.3 and $2305.4 \mathrm{~m} . \mathrm{s}^{-1}$ respectively [26]. Besides, melting temperature $T_{m}$ is also affected by reducing the size that can be found by [38]:

$\frac{T_{m}(r)}{T_{m}(\infty)}=\left(\frac{V(r)}{V(\infty)}\right)^{2 / 3} \exp \left[-\frac{2\left(S_{m}(\infty)-R\right)}{3 R\left(\frac{r}{r_{c}}-1\right)}\right]$

where the melting temperature for bulk magnetite is $1870 \mathrm{~K}$ [39]; $S_{m}(\infty)$ is the value of enthalpy of melting for bulk $\mathrm{Fe}_{3} \mathrm{O}_{4}$, which is equal to $74.61 \mathrm{~J} \cdot K^{-1} \cdot \mathrm{mol}^{-1}[40] ; R$ is ideal gas constant $\left(=8.314 \mathrm{~J} \cdot K^{-1} \cdot \mathrm{mol}^{-1}\right)$ [17]; $r_{c}$ is the critical radius of nano-material at absolute zero. The value of $r_{c}$ is equal to $3 h$, where $h$ denotes the $1^{\text {st }}$ solid surface layer high that that is equal to $1.429 d_{\text {mean }}$, where $d_{\text {mean }}$ is the mean bond length. Also, volume for lattice depends on the size, which can be obtained by:

$V(r)=a^{3}(r)$, 
Equation (32) shows that volume per atom depends on nanosize lattice parameters, $a(r)$, which can be defined as [41]:

$a(r)=\frac{4}{\sqrt{3}} d_{\text {mean }}(r)$

Normally, lattice parameters can be obtained indirectly through x-ray diffraction analysis [42], and hence, by replacing in Equation (32) the bulk volume per atom can be calculated for magnetite. Also, the mean bond length can be estimated by the following equations in [41]:

$d_{\text {mean }}(r)=h-\Delta d_{\text {mean }}\left(r_{c}\right) \quad$ For thin film

$d_{\text {mean }}(\infty)=h-\Delta d_{\text {mean }}\left(r_{c}\right) \quad$ For bulk.

Equation (34) can be rewritten in term of change in mean bond length $\left(\Delta d_{\text {mean }}\right)$, gas constant, critical radius, and the diameter of the thin film [43]:

$d_{\text {mean }}(r)=\Delta d_{\text {mean }}\left(r_{c}\right) \exp \left[\frac{-2 S_{m}(\infty)-R}{3 R\left(\frac{r}{r_{c}}-1\right)}\right]^{1 / 2}$

where $\Delta d_{\text {mean }}\left(r_{c}\right)$ represents the maximum growth in the mean bond length. The critical radius for thin film, where all atoms concentrated on the surface and its value can be obtained by [44]:

$r_{c}=(3-D) h$

where $D$ is the dimension (for thin film, nanowire, and quantum dot are 2, 1, and 0 , respectively [45]; $h$ can be found by [36]:

$h=1.429 d_{\text {mean }}(\infty)$

Furthermore, the enthalpy of fusion is equal to $122.9\left(\mathrm{~kJ}^{\mathrm{mol}}{ }^{-1}\right)$ for $\mathrm{Fe}_{3} \mathrm{O}_{4}$ [46].

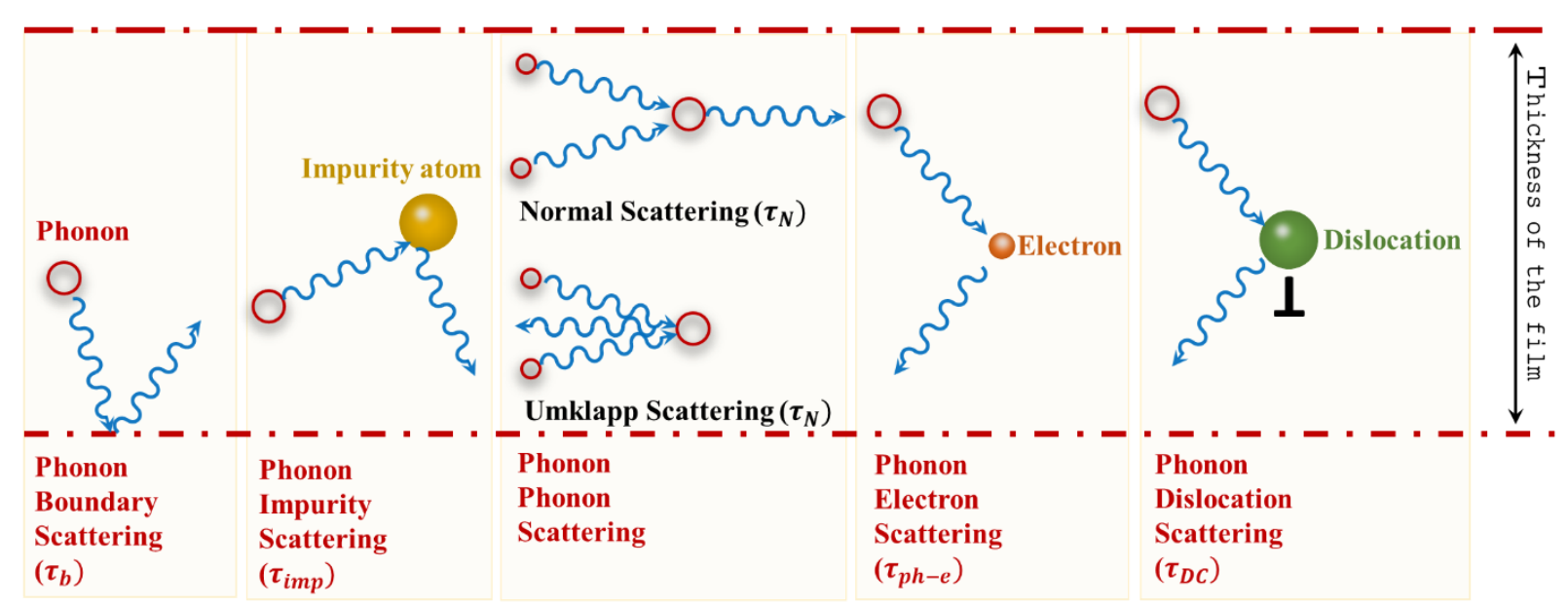

Figure 1. Schematic representation of different phonon scattering in the $\mathrm{Fe}_{3} \mathrm{O}_{4}$ thin films

\section{RESULTS AND DISCUSSION}

In this study, a modified Callaway model has been used to simulate LTC, calculate some physical parameters, and estimate some quantities, which hard to find out with an experimental method. Figure 1 
shows different phonon scattering mechanisms [47] that could happen in the magnetite thin film. The LTC of the thin films were obtained by Equation (2) for the entire range of temperature. Figure 2 shows the LTC for magnetite thin films and bulk. The calculated LTC-based on Modified-Callaway Model has a good agreement with the experimental results in both low and high temperature. In the bulk $\mathrm{Fe}_{3} \mathrm{O}_{4}$, the plot of thermal conductivity against temperature shows a bell-like shape, which has a peak in low temperatures. The peak became more wideness in the nanosized magnetite and the magnetite-thin film with a diameter of $100 \mathrm{~nm}$ the LTC approximately reach a plateau.

Both iron and oxygen have isotopes with different abundance in nature. Table 1 lists four isotopes for Fe and three isotopes for $\mathrm{O}$ that are reported in the literature. Also, by using Equations (36), (33), (32), (31), (30), (29), and (28) each $d_{\text {mean }}$, lattice parameter, volume, melting temperature, Debye temperature, group velocity, and mass density of magnetite thin films were predicted, which are different from the bulk state of the material (Table 2). Additionally, to fit the investigated model some parameters, including impurity, dislocation, electron concentration, gruneisen, and surface roughness was found and listed in Table 3.

Table 1. Material parameters for the $\mathrm{Fe}_{3} \mathrm{O}_{4}$ thin films

\begin{tabular}{lllc}
\hline \hline & Isotope & Value & Reference \\
\hline \hline Fe isotopes abundance & ${ }^{58} \mathrm{Fe}$ & $0.282 \%$ & {$[48]$} \\
& ${ }^{57} \mathrm{Fe}$ & $2.119 \%$ & \\
& ${ }^{56} \mathrm{Fe}$ & $91.754 \%$ & {$[48]$} \\
& ${ }^{54} \mathrm{Fe}$ & $5.845 \%$ & \\
\hline Fe isotopic mass & ${ }^{58} \mathrm{Fe}$ & $57.9332744 \mathrm{amu}$ & \\
& ${ }^{57} \mathrm{Fe}$ & $56.9353928 \mathrm{amu}$ & {$[49]$} \\
& ${ }^{56} \mathrm{Fe}$ & $55.9349363 \mathrm{amu}$ & \\
\hline Oxygen isotopic abundance & ${ }^{54} \mathrm{Fe}$ & $53.9396090 \mathrm{amu}$ & {$[50]$} \\
& ${ }^{18} \mathrm{O}$ & $0.205 \%$ & {$[51]$} \\
\hline${ }^{17} \mathrm{O}$ & $0.038 \%$ & \\
\hline Oxygen isotopes mass & ${ }^{16} \mathrm{O}$ & $99.757 \%$ & $17.9991596128 \mathrm{amu}$ \\
& ${ }^{18} \mathrm{O}$ & $16.9991317566 \mathrm{amu}$ & \\
& ${ }^{17} \mathrm{O}$ & $15.99491461960 \mathrm{amu}$ & \\
\hline
\end{tabular}

Table 2. Calculated mean path length, lattice parameter, lattice volume, mass density, melting temperature, Debye temperatures, and phonon group velocity for the longitudinal and transverse mode of the $\mathrm{Fe}_{3} \mathrm{O}_{4}$ thin films

\begin{tabular}{cccccccccc}
\hline \hline$r$ & $d_{\text {mean }}$ & $\alpha$ & $\mathrm{V}$ & $\rho$ & $T_{m}$ & $\theta_{D}^{L}$ & $\theta_{D}^{T}$ & $v_{L}$ & $v_{T}$ \\
$\mathrm{~nm}$ & $\AA$ & $\AA$ & $\AA^{3}$ & $\mathrm{~kg} \cdot \mathrm{m}^{-3}$ & $\mathrm{~K}$ & $\mathrm{~K}$ & $\mathrm{~K}$ & $\mathrm{~m} / \mathrm{s}$ & $\mathrm{m} / \mathrm{s}$ \\
\hline \hline 100 & 3.67939 & 0.84972 & 613.5 & 4991 & 1811 & 112.059 & 79.1655 & 3159.4 & 2232 \\
300 & 3.65079 & 0.84311 & 599.3 & 5109 & 1850 & 114.522 & 80.9057 & 3228.9 & 2281 \\
400 & 3.64721 & 0.84229 & 597.6 & 5124 & 1855 & 114.828 & 81.1218 & 3237.5 & 2287 \\
Bulk & 3.63644 & $8.39^{\mathrm{b}}$ & $592.3^{\mathrm{c}}$ & 5170 & $1870^{\mathrm{b}}$ & 115.743 & 81.7682 & $3,263.3^{\mathrm{d}}$ & $2,305.4^{\mathrm{d}}$ \\
\hline \hline
\end{tabular}

Table 3. The fitting parameters for the $\mathrm{Fe}_{3} \mathrm{O}_{4}$ thin films

\begin{tabular}{lcccccccc}
\hline \hline$r$ & $N_{\text {imp }}$ & $\begin{array}{c}N_{D} \\
\mathrm{~mm}\end{array}$ & $\begin{array}{c}n_{e} \\
\mathrm{~m}^{-3}\end{array}$ & $\varepsilon$ & $\begin{array}{c}L_{\mathrm{C}} \\
\mathrm{nm}\end{array}$ & $\begin{array}{c}L \\
\mathrm{~mm}\end{array}$ & $\gamma_{L}$ & $\gamma_{T}$ \\
\hline \hline 100 & $5 \times 10^{23}$ & $3 \times 10^{14}$ & $1 \times 10^{23}$ & 0.5 & 100 & 3 & 1.8 & 0.8 \\
300 & $1 \times 10^{23}$ & $1 \times 10^{14}$ & $9 \times 10^{22}$ & 0.7 & 300 & 3 & 0.4 & 0.2 \\
400 & $5 \times 10^{22}$ & $5 \times 10^{13}$ & $7 \times 10^{22}$ & 0.9 & 400 & 3 & 0.3 & 0.08 \\
\hline \hline
\end{tabular}

Figure 2 displays the theoretical and experimental LTC of $\mathrm{Fe}_{3} \mathrm{O}_{4}$ thin films with a thickness of 100, 300, and $400 \mathrm{~nm}$. It is known that an experiment cannot give an exact value, especially for the entire range of measurement, therefore, there are shifts in the experimental data. The Modified-Callaway Model could fit 
the experimental data better than the study conducted by Park et al. [26]. The reason could be the presence of more compounds in the Modified Callaway Model, for example, the Modified Callaway Model is split into two longitudinal and transverse modes, and also the influence of reducing thickness for all sub deviation parameters, including mean bond length, lattice constant, volume per atom, melting temperature, group velocity, and Debye temperature have been taken into account. The thermal conductivity of magnetite thin films is decreased with reducing the thickness of the film.

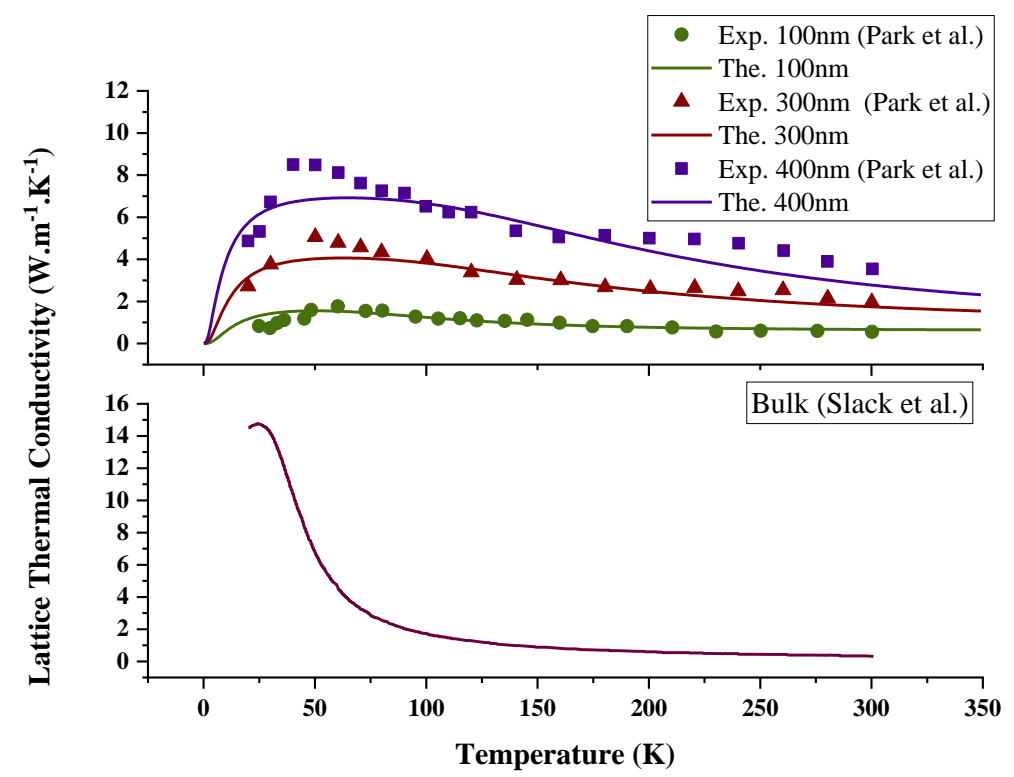

Figure 2. The lattice thermal conductivity of magnetite thin films compared with the corresponding experimental data and bulk $\mathrm{Fe}_{3} \mathrm{O}_{4}$

The calculated parameters are shown in Figure 3. The value of mean bond length, the lattice constant, and volume per atom increased by reducing the thickness of the films while melting temperature is decreased because the interatomic distance is increased that weaknesses the bond. Also, the ultimate vibrational frequency is reached in a lower temperature, which is represented by Debye temperature. Additionally, by reducing the density, the traveling phonon in the solid phase is also decreased. In Figure 3, the quantitative value of the bulk magnetite is presented to be compared with that of thin films. Many searchers, including Abdullah et al. [55] and Omar [56] reported the nano sized effect on the physical parameters and LTC of semiconductor materials. It can be seen that melting temperature is also affected, which means it limits the high temperature applications. However, magnetite normally is not used for temperature above Currie temperature, since it will loss magnetic property, but the model does not concern about this parameter.
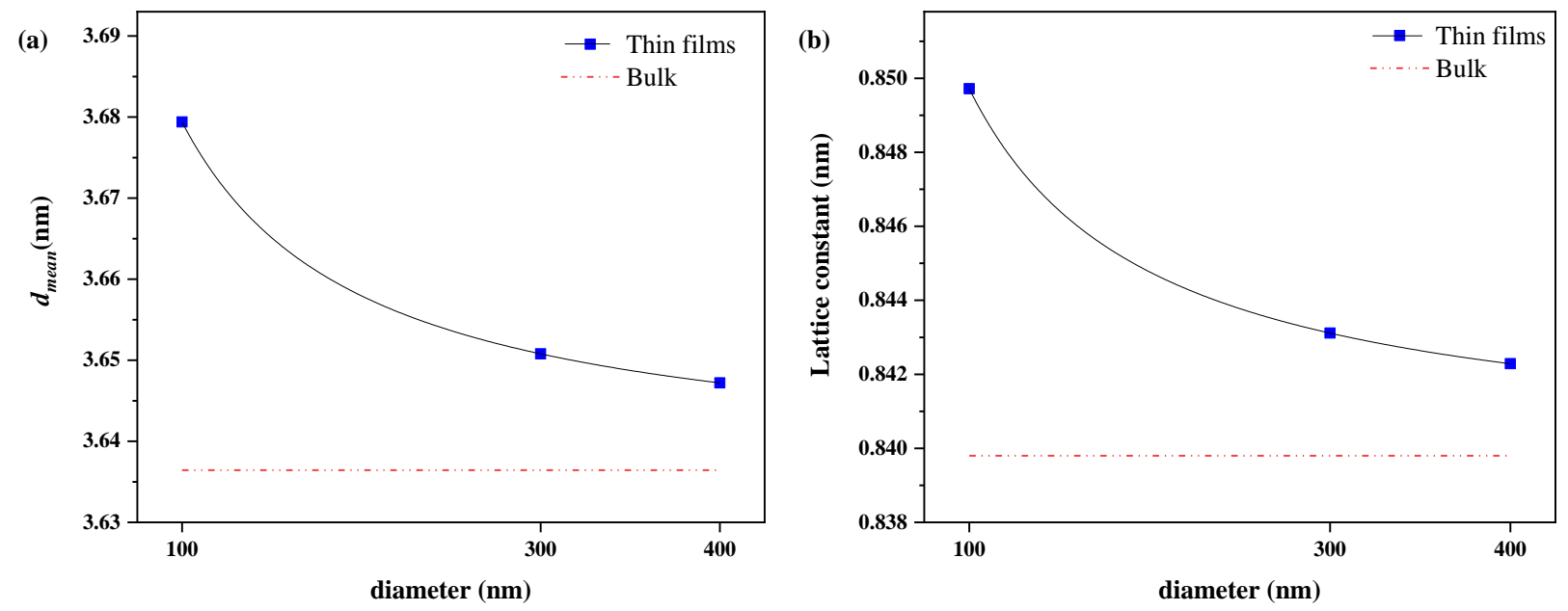

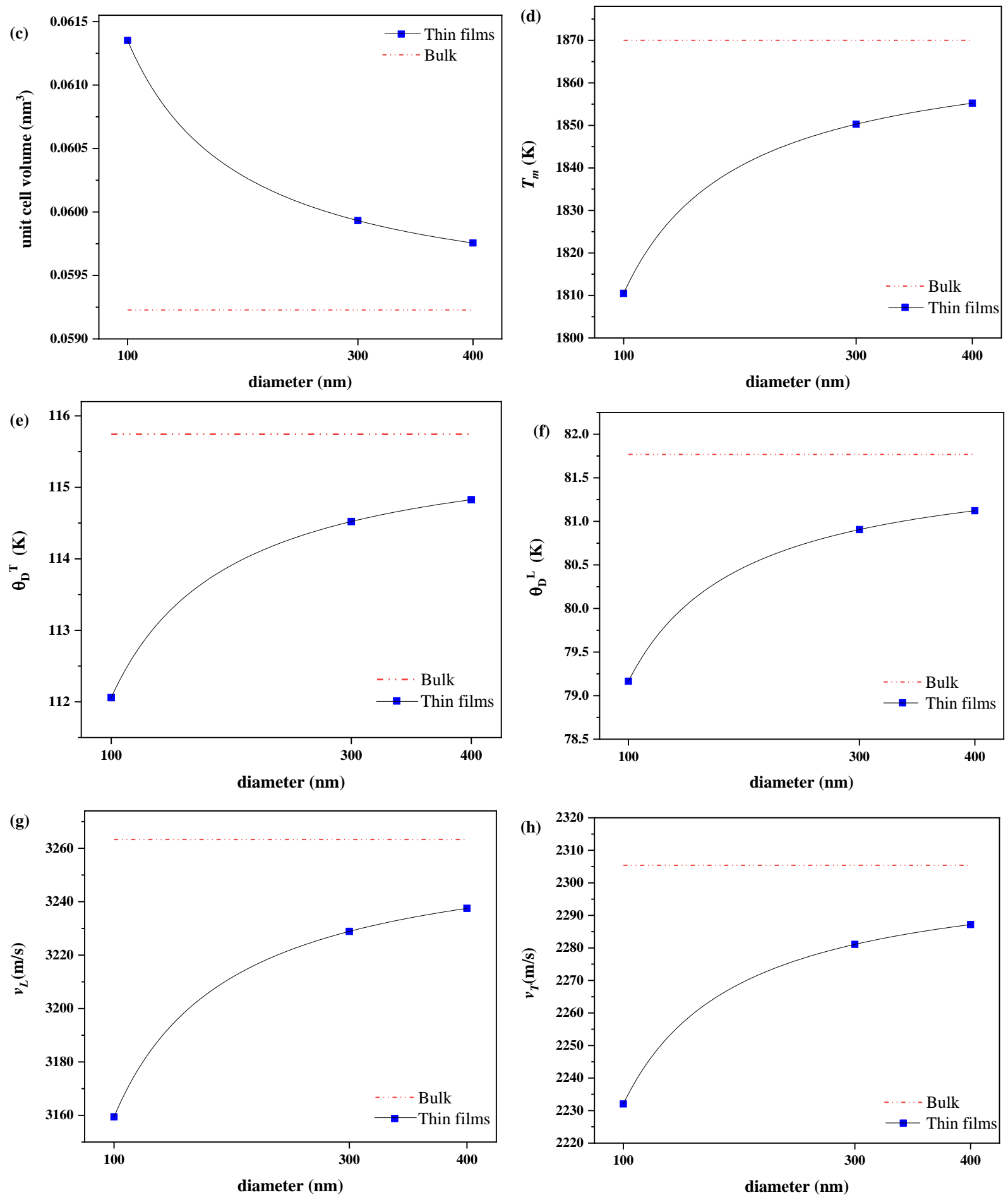

Figure 3. The corresponding nano-sized parameters for $(a) d_{\text {mean, }}(b)$ lattice parameter, $(c)$ volume per atom, $(d)$ melting point, $(e)$ and $(f)$ Debye temperatures, $(g)$ and $(h)$ group velocities

This model makes it promising to find some physical quantities that are hard or impossible to be found with an experimental procedure. Carrier concentration for bulk magnetite reported in the literature is $1.35 \times 10^{-22} \mathrm{~cm}^{-3}$ [57], which is less than the obtained results for thin files. Figure 4a displays that the quantitative value of the carrier concentration diminished by increasing thickness of the film. Besides, dislocation and impurities increased with reducing thickness of the films. One of the disadvantages of this model is that it does not take into account the temperature factor, e.g., the dislocation density can change with temperature [58]. Additionally, Grüneisen parameter, which describe the impact of changing volume on vibration feature, is increased with decreasing the size, while the surface roughness is increased with 
reducing the thickness of the film (Figure 4b). Karim and his colleague also found that the Grüneisen parameter increased with reducing InAs nanowire [19].
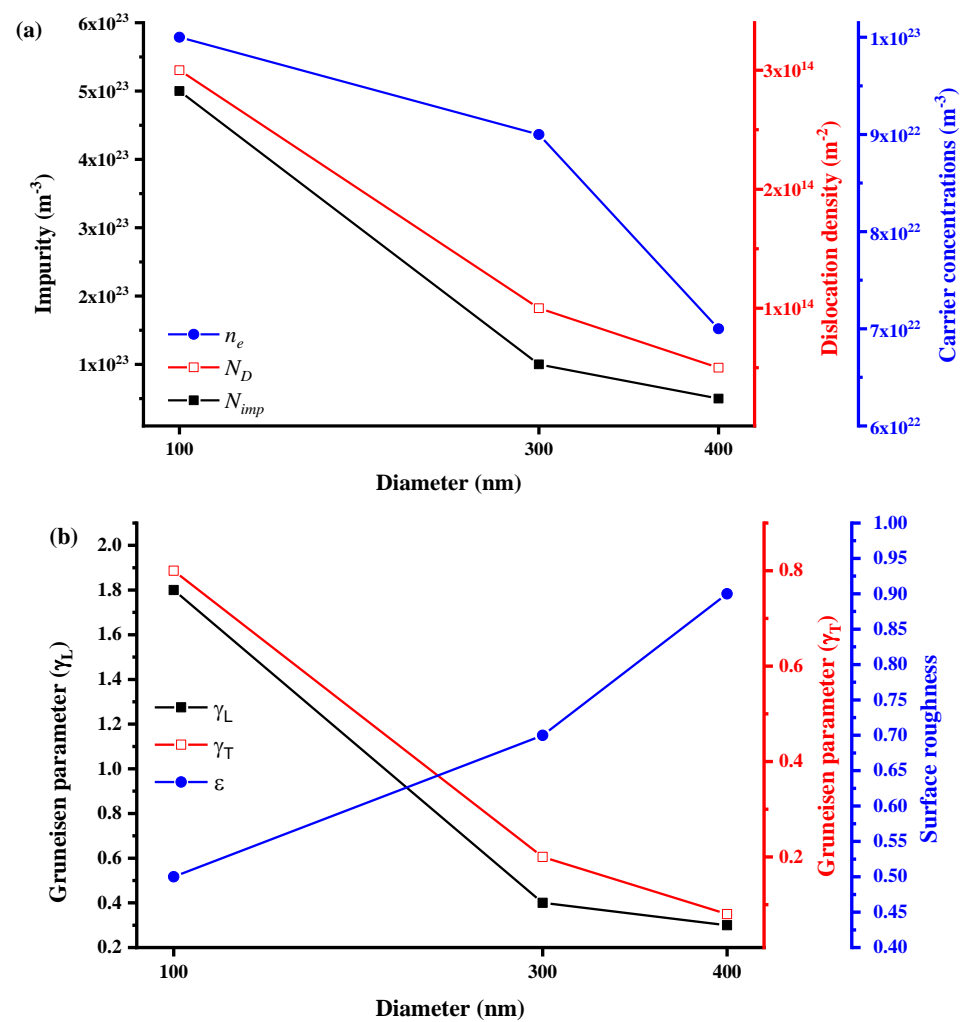

Figure 4. Parameters obtained from fitting the theoretical with experimental LTC for magnetite nonofilms. (a) Impurity, dislocation density, carrier concentration, (b) gruneisen parameters, and surface roughness for three $\mathrm{Fe}_{3} \mathrm{O}_{4}$ thin films

\section{CONCLUSION}

Magnetite $\left(\mathrm{Fe}_{3} \mathrm{O}_{4}\right)$ is an important compound material produced by iron oxide, i.e., $\mathrm{Fe}_{3} \mathrm{O}_{4}$ consists of two elements, which produced an identical material with unique behaviors. They are using in many applications, and nowadays, scientists investigate the physical characteristics of magnetite material in low dimensions. Lattice thermal conductivity (LTC) has a significant role in miniature applications. In this study, the Modified Callaway Model was utilized to anticipate the experimental LTC data of $\mathrm{Fe}_{3} \mathrm{O}_{4}$ for thin films with a thickness of 100,300, and $400 \mathrm{~nm}$. The model could fit data better than the simplified Callaway Model since many physical parameters were taken into account. By using the existing data in the literature for bulk $\mathrm{Fe}_{3} \mathrm{O}_{4}$ and the mathematical equations specific to nanomaterials, we could obtain the corresponding quantities for the respective thin films. Additionally, this model also allowed us to get some values, such as impurity, gruneisen parameter, and dislocations that are actually out of reach by experiment. Consequently, the model could successfully run over the range of temperature of $0.1-350 \mathrm{~K}$ and except for some particular points, it could anticipate experimental LTC.

\section{CONFLICTS OF INTEREST}

No conflict of interest was declared by the authors.

\section{REFERENCES}

[1] Kim, Y. S., Kim, Y. H., "Application of ferro-cobalt magnetic fluid for oil sealing", Journal of Magnetism and Magnetic Materials, 267(1): 105-110, (2003). 
[2] Raj, K., Moskowitz, R., "A review of damping applications of ferrofluids", IEEE Transactions on Magnetics, 16(2): 358-363, (1980).

[3] McMichael, R., Shull, R., Swartzendruber, L., Bennett, L., Watson, R., "Magnetocaloric effect in superparamagnets", Journal of Magnetism and Magnetic Materials, 111(1-2): 29-33, (1992).

[4] Cao, J., Wang, Y., Yu, J., Xia, J., Zhang, C., Yin, D., Häfeli, U. O., "Preparation and radiolabeling of surface-modified magnetic nanoparticles with rhenium-188 for magnetic targeted radiotherapy", Journal of Magnetism and Magnetic Materials, 277(1-2): 165-174, (2004).

[5] Shen, L., Laibinis, P. E., Hatton, T. A., "Bilayer surfactant stabilized magnetic fluids: synthesis and interactions at interfaces", Langmuir, 15(2): 447-453, (1999).

[6] Jordan, A., Scholz, R., Wust, P., Fähling, H., Felix, R., "Magnetic fluid hyperthermia (MFH): Cancer treatment with AC magnetic field induced excitation of biocompatible superparamagnetic nanoparticles", Journal of Magnetism and Magnetic Materials, 201(1-3): 413-419, (1999).

[7] Al Rahal Al Orabi R., Hwang, J., Lin, C.-C., Gautier, R., Fontaine, B., Kim, W., Rhyee, J.-S., Wee, D., Fornari, M., "Ultralow lattice thermal conductivity and enhanced thermoelectric performance in SnTe: Ga materials", Chemistry of Materials, 29(2): 612-620, (2017).

[8] Dong, J., Sun, F.H., Tang, H., Hayashi, K., Li, H., Shang, P.P., Miyazaki, Y., Li, J.F., "Reducing lattice thermal conductivity of MnTe by Se alloying toward high thermoelectric performance", ACS applied materials \& interfaces, 11(31): 28221-28227, (2019).

[9] Blaney, L., Magnetite (Fe3O4): Properties, synthesis, and applications, Pennsylvania, 36-81, (2007).

[10] Lian, S., Kang, Z., Wang, E., Jiang, M., Hu, C., Xu, L., "Convenient synthesis of single crystalline magnetic Fe3O4 nanorods", Solid State Communications, 127(9-10): 605-608, (2003).

[11] Jha, M. K., Kumar, V., Maharaj, L., Singh, R., "Studies on leaching and recycling of zinc from rayon waste sludge", Industrial \& engineering chemistry research, 43(5): 1284-1295, (2004).

[12] Xie, J., Xu, C., Kohler, N., Hou, Y., Sun, S., "Controlled PEGylation of monodisperse Fe3O4 nanoparticles for reduced non-specific uptake by macrophage cells", Advanced Materials, 19(20): 3163-3166, (2007).

[13] Sun, S., Zeng, H., "Size-controlled synthesis of magnetite nanoparticles", Journal of the American Chemical Society, 124(28): 8204-8205, (2002).

[14] Sun, S., Murray, C. B., Weller, D., Folks, L., Moser, A., "Monodisperse FePt nanoparticles and ferromagnetic FePt nanocrystal superlattices", Science, 287(5460): 1989-1992, (2000).

[15] Shen, Y., Tang, J., Nie, Z., Wang, Y., Ren, Y., Zuo, L., "Preparation and application of magnetic Fe3O4 nanoparticles for wastewater purification", Separation and Purification Technology, 68(3): 312-319, (2009).

[16] Pankhurst, Q. A., Connolly, J., Jones, S. K., Dobson, J., "Applications of magnetic nanoparticles in biomedicine", Journal of physics D: Applied physics, 36(13): R167, (2003).

[17] Qader, I. N., Omar, M. S., "Carrier concentration effect and other structure-related parameters on lattice thermal conductivity of Si nanowires", Bulletin of Materials Science, 40(3): 599-607, (2017). 
[18] Mamand, S. M., Omar, M. S., "Effect of Parameters on Lattice Thermal Conductivity in Germanium Nanowires", Advanced Materials Research, 832: 33-38, (2014).

[19] Karim, H. H., Omar, M. S., "Temperature-dependence calculation of lattice thermal conductivity and related parameters for the zinc blende and wurtzite structures of InAs nanowires", Bulletin of Materials Science, 43(1): 54, (2020).

[20] Mamand, S.M., Omar, M.S., Muhammed, A.J., "Calculation of lattice thermal conductivity of suspended GaAs nanobeams: Effect of size dependent parameters", Advanced Materials Letters, 3(6): 449-458, (2012).

[21] Mamand, S. M., Omar, M. S., Muhammad, A. J., "Nanoscale size dependence parameters on lattice thermal conductivity of Wurtzite GaN nanowires", Materials Research Bulletin, 47(5): 1264-1272, (2012).

[22] Asen-Palmer, M., Bartkowski, K., Gmelin, E., Cardona, M., Zhernov, A. P., Inyushkin, A. V., Taldenkov, A., Ozhogin, V. I., Itoh, K. M., Haller, E. E., "Thermal conductivity of germanium crystals with different isotopic compositions", Physical Review B, 56(15): 9431-9447, (1997).

[23] Omar, M., Taha, H., "Effects of nanoscale size dependent parameters on lattice thermal conductivity in Si nanowire", Sadhana, 35(2): 177-193, (2010).

[24] Qader, I. N., Abdullah, B. J., Omar, M. S., "Range Determination of the Influence of Carrier Concentration on Lattice Thermal Conductivity for Bulk Si and Nanowires", Aksaray University Journal of Science and Engineering, 4(1): 30-42, (2020).

[25] Su, J., Liu, Z.-t., Feng, L.-p., Li, N., "Effect of temperature on thermal properties of monolayer MoS2 sheet", Journal of alloys and compounds, 622: 777-782, (2015).

[26] Park, N.-W., Lee, W.-Y., Kim, J.-A., Song, K., Lim, H., Kim, W.-D., Yoon, S.-G., Lee, S.-K., "Reduced temperature-dependent thermal conductivity of magnetite thin films by controlling film thickness", Nanoscale Research Letters, 9(1): 96, (2014).

[27] Morelli, D. T., Heremans, J. P., Slack, G. A., "Estimation of the isotope effect on the lattice thermal conductivity of group IV and group III-V semiconductors", Physical Review B, 66(19): 195304, (2002).

[28] Klemens, P., "The scattering of low-frequency lattice waves by static imperfections", Proceedings of the Physical Society. Section A, 68(12): 1113, (1955).

[29] Vandersande, J., "Thermal-conductivity reduction in electron-irradiated type-II a diamonds at low temperatures", Physical Review B, 15(4): 2355, (1977).

[30] Zhu, X., Zou, X., Liang, B., Cheng, J., "One-way mode transmission in one-dimensional phononic crystal plates", Journal of Applied Physics, 108(12): 124909, (2010).

[31] Zou, J., Kotchetkov, D., Balandin, A. A., Florescu, D. I., Pollak, F. H., "Thermal conductivity of GaN films: Effects of impurities and dislocations", Journal of Applied Physics, 92(5): 2534-2539, (2002).

[32] Zou, J., Balandin, A., "Phonon heat conduction in a semiconductor nanowire", Journal of Applied Physics, 89(5): 2932-2938, (2001). 
[33] Kumar, A., Pandya, D. K., Chaudhary, S., "Structural, electronic, and magnetic behavior of two dimensional epitaxial Fe3O4/TiN/Si(100) system", Applied Physics Letters, 102(15): 152406, (2013).

[34] Li, Z. W., Yang, Z. H., "Microwave absorption properties and mechanism for hollow Fe3O4 nanosphere composites", Journal of Magnetism and Magnetic Materials, 387: 131-138, (2015).

[35] Pranita, L., Preeti, J., "Preparation and characterization of magnetite nanoparticle using green synthesis", International Journal Of Research In Chemistry And Environment, 5: 38-43, (2015).

[36] Qader, I. N., Abdullah, B. J., Hassan, M. A., Mahmood, P. H., "Influence of the Size Reduction on the Thermal Conductivity of Bismuth Nanowires", Eurasian Journal of Science and Engineering, 4(3): 55-65, (2019).

[37] Abdullah, B. J., Jiang, Q., Omar, M. S., "Effects of size on mass density and its influence on mechanical and thermal properties of $\mathrm{ZrO} 2$ nanoparticles in different structures", Bulletin of Materials Science, 39(5): 1295-1302, (2016).

[38] Karim, H. H., Omar, M., "Temperature-dependence calculation of lattice thermal conductivity and related parameters for the zinc blende and wurtzite structures of InAs nanowires", Bulletin of Materials Science, 43(1): 54, (2020).

[39] Blaney, L., "Magnetite (Fe3O4): Properties, synthesis, and applications", Lehigh Reveview, 15: 32-81, (2007).

[40] Moskvin, P. P., Olchowik, J. M., "Model of polyassosiative solutions and its application for the analysis of $\mathrm{p}-\mathrm{T}-\mathrm{x}$ equilibrium in iron-like oxide solutions and A2B6 semiconductor systems", Journal of Crystal Growth, 361: 98-102, (2012).

[41] Omar, M., "Structural and Thermal Properties of Elementary and Binary Tetrahedral Semiconductor Nanoparticles", International Journal of Thermophysics, 37(1): 11, (2016).

[42] Imran, M., Akbar, A., Riaz, S., Atiq, S., Naseem, S., "Electronic and Structural Properties of PhasePure Magnetite Thin Films: Effect of Preferred Orientation", Journal of Electronic Materials, 47(11): 6613-6624, (2018).

[43] Jiang, Q., Yang, C., "Size effect on the phase stability of nanostructures", Current nanoscience, 4(2): 179-200, (2008).

[44] Qader, I. N., Abdullah, B. J., Karim, H. H., "Lattice Thermal Conductivity of Wurtzite Bulk and Zinc Blende CdSe Nanowires and Nanoplayer", Eurasian Journal of Science \& Engineering, 3(1): 9-26, (2017).

[45] Omar, M. S., "Models for mean bonding length, melting point and lattice thermal expansion of nanoparticle materials", Materials Research Bulletin, 47(11): 3518-3522, (2012).

[46] Sahebari, M., Raigan, S., Seyed, E. S., Abdizadeh, H., "Inception of transformation of hematite to magnetite during mechanical activation: a thermodynamical approach", Iranian Journal of Science and Technology Transaction B- Engineering, 33(85), (2009).

[47] Vasileska, D., Hossain, A., Raleva, K., Goodnick, S. M., "The role of the source and drain contacts on self-heating effect in nanowire transistors", Journal of Computational Electronics, 9(3): 180186, (2010). 
[48] Poitrasson, F., Iron, Isotopes.In: M. Gargaud, R. Amils, J. C. Quintanilla, H. J. Cleaves, W. M. Irvine, D. L. Pinti and M. Viso, editors., Berlin, Heidelberg. 852-855 Encyclopedia of Astrobiology. Springer Berlin Heidelberg; (2011).

[49] Wright, L. E., Oxygen Isotopes.In: A. S. Gilbert, editor., Dordrecht. 567-574 Encyclopedia of Geoarchaeology. Springer Netherlands; (2017).

[50] Audi, G., Wapstra, A. H., Thibault, C., "The Ame2003 atomic mass evaluation: (II). Tables, graphs and references", Nuclear Physics A, 729(1): 337-676, (2003).

[51] Velichko, T. I., Mikhailenko, S. N., Tashkun, S. A., "Global Multi-isotopologue fit of measured rotation and vibration-rotation line positions of $\mathrm{CO}$ in X1 $\Sigma+$ state and new set of mass-independent Dunham coefficients", Journal of Quantitative Spectroscopy and Radiative Transfer, 113(13): 1643-1655, (2012).

[52] Heiße, F., Köhler-Langes, F., Rau, S., Hou, J., Junck, S., Kracke, A., Mooser, A., Quint, W., Ulmer, S., Werth, G., "High-precision measurement of the proton's atomic mass", Physical Review Letters, 119(3): 033001, (2017).

[53] Fei, Y., Frost, D. J., Mao, H.-K., Prewitt, C. T., Haeusermann, D., "In situ structure determination of the high-pressure phase of Fe3O4", American Mineralogist, 84(1-2): 203-206, (1999).

[54] Park, N.-W., Lee, W.-Y., Kim, J.-A., Song, K., Lim, H., Kim, W.-D., Yoon, S.-G., Lee, S.-K., "Reduced temperature-dependent thermal conductivity of magnetite thin films by controlling film thickness", Nanoscale research letters, 9(1): 1-8, (2014).

[55] Abdullah, B. J., Omar, M. S., Jiang, Q., "Size dependence of the bulk modulus of Si nanocrystals", Sadhana, 43(11): 174, (2018).

[56] Omar, M. S., "Structural and Thermal Properties of Elementary and Binary Tetrahedral Semiconductor Nanoparticles", International Journal of Thermophysics, 37(1): 11, (2016).

[57] Shchennikov, V. V., Ovsyannikov, S. V., Karkin, A. E., Todo, S., Uwatoko, Y., "Galvanomagnetic properties of fast neutron bombarded $\mathrm{Fe} 3 \mathrm{O} 4$ magnetite: A case against charge ordering mechanism of the Verwey transition", Solid State Communications, 149(19): 759-762, (2009).

[58] Callister, W. D., An Introduction: Material Science and Engineering. New York, 106-139, 2007. 